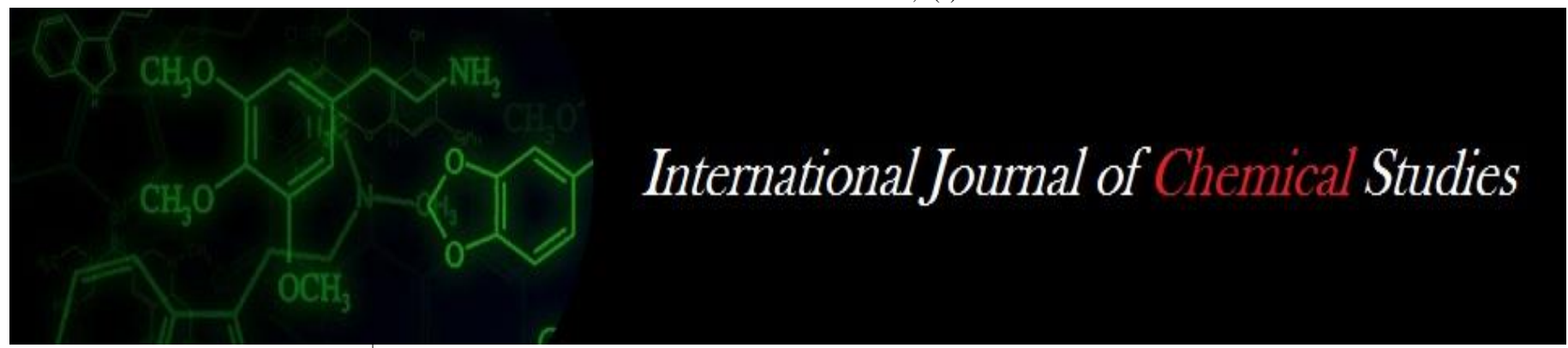

P-ISSN: 2349-8528

E-ISSN: 2321-4902

www.chemijournal.com

IJCS 2020; 8(2): 2708-2713

(C) 2020 IJCS

Received: 15-01-2020

Accepted: 19-02-2020

Gabu Singh Gathiye

Scientist (Agronomy),

Krishi Vigyan Kendra, Dhar,

Madhya Pradesh, India

Vishal Verma

M.Sc. Scholar, Deptt. of Genetic and Plant Breeding,

Bundelkhand University, Jhansi,

Uttar Pradesh, India
Corresponding Author: Gabu Singh Gathiye Scientist (Agronomy), Krishi Vigyan Kendra, Dhar, Madhya Pradesh, India

\section{Effect of fungicide and different Rhizobium inoculants on growth and yield of soybean (Glycine max (L.) Merrill)}

\author{
Gabu Singh Gathiye and Vishal Verma
}

DOI: https://doi.org/10.22271/chemi.2020.v8.i2ao.9159

\begin{abstract}
An experiment was carried out at in Kharif season at student research field, College of Agriculture, Indore (M.P.) to study the Effect of fungicide and different Rhizobium inoculants on growth and yield of soybean (Glycine max (L.) Merrill). The soil of the experimental field was medium black in texture, neutral in reaction $(\mathrm{pH} 7.70)$ with normal EC $(0.23 \mathrm{dS} / \mathrm{m})$ and medium organic carbon contents $(0.56 \%)$ and analysing low in available $\mathrm{N}(225 \mathrm{~kg} / \mathrm{ha})$, medium in available $\mathrm{P}(9.60 \mathrm{~kg} / \mathrm{ha})$ and high in available $\mathrm{K}$ $(508 \mathrm{~kg} / \mathrm{ha})$ contents. A field experiment was consisted of 15 treatments replicated four times in randomized block design (RBD). As per treatment, the seed of soybean cv. JS 335 treated or not treated with fungicides and inoculated with Rhizobium culture before 15, 10, 05, 01 days of planting and on the day of sowing. Among the treatments, seed treatment with Thiram + Carbendazim $50 \mathrm{WP}$ - inoculation with Premax+ Rizo-liq (ODS)] ( $\left.\mathrm{T}_{10}\right)$ recorded the maximum plant height $(66.75 \mathrm{~cm})$ at harvest, branches per plant (4.02), fresh weight $(35.83 \mathrm{~g})$ per plant at $75 \mathrm{DAS}$, dry matter per plant $(23.14 \mathrm{~g})$ at $75 \mathrm{DAS}$, leaf area index (12.87) at 60 DAS, number of pods (39.18) per plant, number of seeds (2.84) per pod while minimum was recorded in $\mathrm{T}_{15}$ absolute control (No fungicide, No culture). The maximum yield $(2.15 \mathrm{t} / \mathrm{ha})$ was recorded under [Thiram + Carbendazim 50 WP - Premax+ Rizo-liq (ODS)] $\left(\mathrm{T}_{10}\right)$ treatment and the minimum $(1.51 \mathrm{t} / \mathrm{ha})$ was recorded in absolute control $\left(\mathrm{T}_{15}\right)$ treatment. The application of Rhizobium with or without fungicides showed a synergic effect in increasing the seed yield of soybean per hectare.
\end{abstract}

Keywords: Soybean, fungicide, Bradyrhizobium japonicum (Premax + Rizo-liq), seed treatment, leaf area index and yield

\section{Introduction}

Soybean (Glycine max (L.) Merrill) is a major legume crop recognized as the efficient producer of the two scarce quality characters i.e. the protein and oil, which are not only the major components in the diet of vegetarians mass but a boon to the developing countries as well. Soybean contains 40 per cent protein, 20 per cent oil, 85 per cent PUFA and 25-30 per cent, carbohydrates, minerals, antioxidants, beta-carotene and iso-flavanoids. Soybean plays a vital role in the agricultural economy of India. Soybean [Glycine max (L.) Merrill] is one of the major economically predominant oilseed crops. It is recognized as the most important versatile and fascinating crop of the world. Hence it is known as a "Wonder crop" and "Golden bean" of the $21^{\text {st }}$ century. Soybean is rich in a lysine an amino acid in which most of the cereals are deficit.

In Madhya Pradesh, Farmers generally apply unbalanced under dose of fertilizers and less use of FYM and bacterial cultures which lead to low production. Indiscriminate use of chemical fertilizers deteriorates the soil health with environmental pollution. Biofertilizers are the substitute or supplementary materials in addition to the chemical fertilizers. Biofertilizers are economically viable lever for realizing the ultimate goal of increasing productivity. These microbial systems siphon out appreciable amount of nitrogen from the atmospheric reservoir and enrich the soil with these important but scare nutrients. Culture inoculation of legume seeds at the time of sowing was found helpful in increasing the Rhizobia population in the soil which resulted into increased number of root nodules and ultimately gave 20-70\% more yield of the legume (Dadson and Acquash, 1984) ${ }^{[5]}$ 
The limitation of using the Rhizobia are that they cannot apply well in advance and in the other hand there is narrow window of soybean planting in India as it is rainy season crop. Soybean is becoming popular in Madhya Pradesh particularly in 'Malwa region' and hence efforts should be made to boost up the production of soybean by adopting modern techniques of crop production. The genuine problems limiting the production of soybean are poor germination and low seed viability. Micro-organisms play major role on quality of seed, of which fungi are in predominant quantity. These fungi are harmful as they minimize the vigour of seed and diminish the growth of plant at its initial stages. Fungicides or microbial antagonists act as a barrier for seed infection and seed treating with these save the seed from infection by seed borne and soil borne pathogens. Seed treatment is therefore a routine practice to ensure good emergence and better crop stand. These treatments allow the seed to germinate rapidly in to a healthy seedling. The present study was aimed to know the effect of seed treatment along with fungicides and bioinoculants on growth and seed yield parameters of soybean.

\section{Material and Methods}

An experiment was carried out at in Kharif season at student research field, College of Agriculture, Indore (M.P.) to study the Effect of fungicide and different Rhizobium inoculants on growth and yield of soybean (Glycine $\max$ (L.) Merrill). The soil of the experimental field was medium black in texture, neutral in reaction ( $\mathrm{pH} 7.70)$ with normal EC $(0.23 \mathrm{dS} / \mathrm{m})$ and medium organic carbon contents $(0.56 \%)$ and analysing low in available $\mathrm{N}(225 \mathrm{~kg} / \mathrm{ha})$, medium in available $\mathrm{P}(9.60$ $\mathrm{kg} / \mathrm{ha})$ and high in available $\mathrm{K}(508 \mathrm{~kg} / \mathrm{ha})$ contents. Due to dominance of montmorillonite clay content it has high capacity to swell and shrink and high CEC. A field experiment was consisted of 15 treatments replicated four times in randomized block design (RBD). It is located on latitude of $22.43^{\circ} \mathrm{N}$ and longitude of $75.66^{\circ} \mathrm{E}$. It has subtropical climate having a temperature range of $23{ }^{\circ} \mathrm{C}$ to 41 ${ }^{\circ} \mathrm{C}$ and $4{ }^{\circ} \mathrm{C}$ to $29{ }^{\circ} \mathrm{C}$ in summer and winter season, respectively. The rainfall in the region is mostly inadequate and erratic. Late commencement, early withdrawal and two to three dry spells are the main features. The average rain is 964 $\mathrm{mm}$ and it was below normal $(803 \mathrm{~mm})$. The maximum temperature ranged from $25.7{ }^{\circ} \mathrm{C}$ to $40.8{ }^{\circ} \mathrm{C}$ while minimum temperature accelerated between $14.9{ }^{\circ} \mathrm{C}$ to $28.8{ }^{\circ} \mathrm{C}$ during the season.

\section{Properties of fungicides and Bio-fertilizers}

Thiram: Thiram is contact fungicide, most effective seed protectant, least phytotoxic and used for the control of many seed-borne or soil-borne diseases.

Carbendazim 50 WP (Bavistin): Carbendazim is systemic with prophylactic and curative action and also nonphytotoxic. It is used for the control of many internally and externally seed borne diseases. Besides the disease control, beneficial side effects like stimulation of growth, flowering and yield of plants on the treated hosts have been reported.

Premax (Protector): Premax protects Rhizobial population from the adverse effects of fungicides.

Rizo-liq: Rizo-liq (Bradyrhizobium japonicum Strain) is a liquid biofertilizer which is used well in advance to inoculate the seed and recommended for soybean crop.
Rhizobium cultures (Rh1, $\left.\mathbf{R} \mathbf{h}_{2}, \mathbf{R} \mathbf{h}_{3}\right)$ : Rhizobium cultures (Rhizobium japonicum strain) are solid biofertilizers which are used to inoculate the seed of soybean.

\section{Pre Harvest Studies}

\section{Height of the plant $(\mathrm{cm})$}

In each net plot five plants were selected randomly and tagged for periodic observation. The height was recorded at 30, 45, 60, 75 DAS and at harvest in all the plots. It was measured from the ground surface to the main stem apex.

\section{Number of branches per plant}

The primary branches were counted on five tagged plants at 45,60 DAS and at harvesting of crop in all the plots.

\section{Fresh weight per Plant (g)}

Fresh weight studies were made in a sample of 5 plants removed from the sampling row randomly from each plot at $45,60,75$ DAS. The fresh weight of the sample was recorded an averaged for fresh weight per plant.

\section{Dry matter per Plant (g)}

The above samples were kept in oven at $65{ }^{\circ} \mathrm{C}$ for 48 hours. The dry weight of the sample was recorded an averaged for dry matter per plant.

\section{Leaf area index (LAI)}

It express the total leaf area in relation with the total ground area in which the crop is grown as calculated by the following equation:

$$
\text { Leaf area index }=\frac{\text { Total leaf area of the crop }}{\text { Total ground area under the crop }}
$$

\section{Post Harvest Studies}

\section{Number of pods per plant}

The number of pods per plant was counted from five already tagged plants and mean per plant was calculated for analysis. The observation taken from all the plots.

\section{Number of grains per pod}

The number of grains per pod was counted from 20 randomly selected pods of 5 tagged plants for each plot.

\section{Seed yield (tone per hectare)}

The seed yield per net plot was recorded after drying the seed. The plot yield was later on converted into $\mathrm{kg}$ per hectare by multiplying it by conversion factor.

\section{Stover yield (tone per hectare)}

The stover yield per plot was obtained by subtracting grain yield from bundle weight of each plot. This was later on converted into $\mathrm{kg}$ per hectare.

\section{Results and Discussion \\ Growth parameters \\ Plant Height (cm)}

Plant height is an important index of plant growth, which usually affects total dry matter production of the crop and differs due to different treatments and agronomic conditions. The maximum (66.75) plant height at harvest was recorded in $\mathrm{T}_{10}$ [Thiram + Carbendazim 50 WP - Premax + Rizo-liq (ODS)] treatment, which was closely $(65.05 \mathrm{~cm})$ followed by $\mathrm{T}_{13}$ [Thiram + Carbendazim $50 \mathrm{WP}-\mathrm{Rh}_{3}$ (ODS)] treatment whereas minimum $(62.15 \mathrm{~cm})$ plant height was recorded in $\mathrm{T}_{15}$ (Absolute Control) treatment. 
Table 1: Effect of different treatments on average plant height at successive stages of growth

\begin{tabular}{|c|c|c|c|c|c|c|}
\hline \multirow{2}{*}{ Tr. No. } & \multirow{2}{*}{ Treatments } & \multicolumn{5}{|c|}{ Plant height (cm) } \\
\hline & & 30 DAS & 45 DAS & 60 DAS & 75 DAS & Harvest \\
\hline $\mathrm{T}_{1}$ & Premax + Rizo-liq (15 DPS) & 26.30 & 39.75 & 54.65 & 64.60 & 63.20 \\
\hline $\mathrm{T}_{2}$ & Premax + Rizo-liq (10 DPS) & 26.60 & 41.05 & 55.35 & 65.15 & 63.75 \\
\hline $\mathrm{T}_{3}$ & Premax + Rizo-liq (05 DPS) & 26.65 & 41.93 & 55.55 & 65.35 & 63.95 \\
\hline $\mathrm{T}_{4}$ & Premax + Rizo-liq (01 DPS) & 26.45 & 42.33 & 58.15 & 65.75 & 64.30 \\
\hline $\mathrm{T}_{5}$ & Premax + Rizo-liq (ODS) & 28.15 & 43.90 & 58.80 & 67.40 & 64.65 \\
\hline $\mathrm{T}_{6}$ & Thiram+Carbendazim 50 WP- Premax + Rizo-liq (15 DPS) & 26.15 & 39.35 & 55.05 & 64.55 & 62.95 \\
\hline $\mathrm{T}_{7}$ & Thiram + Carbendazim 50 WP - Premax + Rizo-liq (10 DPS) & 26.75 & 40.35 & 55.60 & 65.10 & 63.25 \\
\hline $\mathrm{T}_{8}$ & Thiram + Carbendazim 50 WP - Premax + Rizo-liq (05 DPS) & 26.30 & 41.43 & 57.35 & 65.70 & 64.45 \\
\hline $\mathrm{T}_{9}$ & Thiram + Carbendazim 50 WP - Premax + Rizo-liq (01 DPS) & 27.15 & 41.93 & 58.35 & 66.30 & 64.60 \\
\hline $\mathrm{T}_{10}$ & Thiram + Carbendazim 50 WP - Premax + Rizo-liq (ODS) & 29.55 & 44.10 & 61.15 & 69.15 & 66.75 \\
\hline $\mathrm{T}_{11}$ & Thiram+Carbendazim $50 \mathrm{WP}-\mathrm{Rh}_{1}(\mathrm{ODS})$ & 27.73 & 42.25 & 58.40 & 66.80 & 64.60 \\
\hline $\mathrm{T}_{12}$ & Thiram+Carbendazim $50 \mathrm{WP}-\mathrm{Rh}_{2}(\mathrm{ODS})$ & 28.20 & 42.25 & 58.95 & 66.70 & 64.65 \\
\hline $\mathrm{T}_{13}$ & Thiram+Carbendazim 50 WP-Rh 3 (ODS) & 28.60 & 42.75 & 60.90 & 67.68 & 65.05 \\
\hline $\mathrm{T}_{14}$ & Uninoculated seed (but fungicidal treatment) & 25.50 & 38.90 & 54.50 & 64.23 & 62.70 \\
\hline $\mathrm{T}_{15}$ & Absolute control (No fungicidal treatment and no inoculation) & 24.50 & 38.25 & 54.20 & 63.23 & 62.15 \\
\hline SEm \pm & & 0.67 & 0.74 & 0.74 & 0.77 & 0.77 \\
\hline $\mathrm{CD}$ at $5 \%$ & & 1.92 & 2.12 & 2.10 & 2.20 & 2.21 \\
\hline
\end{tabular}

\section{Number of branches per plant}

Maximum number of branches per plant (4.02) was recorded in $\mathrm{T}_{10}$ [Thiram + Carbendazim $50 \mathrm{WP}$ - Premax+Rizo-liq (ODS)] treatment and minimum (2.95) was recorded under $\mathrm{T}_{15}$ (Absolute control) treatment. The data revealed that
Rhizobium inoculation with or without fungicide on the day of sowing increased the number of branches per plant as compared to control and uninoculated seed treatment at all the growth stages. However rest of the treatments produced more number of branches per plant than absolute control.

Table 2: Number of branches per plant as affected by various treatments at successive stages of plant growth

\begin{tabular}{|c|c|c|c|c|}
\hline \multirow{2}{*}{ Treatment No. } & \multirow{2}{*}{ Treatments } & \multicolumn{3}{c|}{ Number of branches per plant } \\
\cline { 3 - 5 } & & 45 DAS & 60 DAS & At Harvest \\
\hline $\mathrm{T}_{1}$ & Premax + Rizo-liq (15 DPS) & 1.65 & 3.10 & 3.10 \\
\hline $\mathrm{T}_{2}$ & Premax + Rizo-liq (10 DPS) & 1.80 & 3.20 & 3.20 \\
\hline $\mathrm{T}_{3}$ & Premax + Rizo-liq (05 DPS) & 1.85 & 3.20 & 3.20 \\
\hline $\mathrm{T}_{4}$ & Premax + Rizo-liq (01 DPS) & 1.90 & 3.80 & 3.80 \\
\hline $\mathrm{T}_{5}$ & Premax + Rizo-liq (ODS) & 2.15 & 3.95 & 3.95 \\
\hline $\mathrm{T}_{6}$ & Thiram + Carbendazim 50 WP - Premax + Rizo-liq (15 DPS) & 1.85 & 3.15 & 3.15 \\
\hline $\mathrm{T}_{7}$ & Thiram + Carbendazim 50 WP - Premax + Rizo-liq (10 DPS) & 1.90 & 3.25 & 3.25 \\
\hline $\mathrm{T}_{8}$ & Thiram + Carbendazim 50 WP - Premax + Rizo-liq (05 DPS) & 1.95 & 3.30 & 3.30 \\
\hline $\mathrm{T}_{9}$ & Thiram + Carbendazim 50 WP - Premax + Rizo-liq (01 DPS) & 2.10 & 3.80 & 3.80 \\
\hline $\mathrm{T}_{10}$ & Thiram + Carbendazim 50 WP - Premax + Rizo-liq (ODS) & 2.60 & 4.02 & 4.02 \\
\hline $\mathrm{T}_{11}$ & Thiram + Carbendazim 50 WP - Rh (ODS) & 2.20 & 3.85 & 3.85 \\
\hline $\mathrm{T}_{12}$ & Thiram + Carbendazim 50 WP - Rh $_{2}$ (ODS) & 2.25 & 3.80 & 3.80 \\
\hline $\mathrm{T}_{13}$ & Thiram + Carbendazim 50 WP - Rh 3 (ODS) & 2.55 & 3.95 & 3.95 \\
\hline $\mathrm{T}_{14}$ & Uninoculated seed (but fungicidal treatment) & 1.60 & 3.05 & 3.05 \\
\hline $\mathrm{T}_{15}$ & Absolute control & 1.55 & 2.95 & 2.95 \\
\hline SEm \pm & (No fungicidal treatment and no inoculation) & 0.23 & 0.31 & 0.31 \\
\hline $\mathrm{CD}$ at $5 \%$ & & $\mathrm{NS}$ & $\mathrm{NS}$ & $\mathrm{NS}$ \\
\hline
\end{tabular}

DPS: Days prior sowing, ODS: On days of sowing, DAS: Days after sowing

\section{Fresh weight per plant (g)}

Data revealed that fresh weight per plant increased with advancement in age of the plant. It was found that rate of increase in fresh weight was maximum between 60 to 75 DAS in almost all the treatments and minimum between 45 to 60
DAS.The treatment $\mathrm{T}_{10}$ [Thiram + Carbendazim $50 \mathrm{WP}$ Premax + Rizo-liq (ODS)] resulted in 16.44, 22.6 and $35.83 \mathrm{~g}$ fresh weight per plant at 45, 60 and 75 DAS, respectively which were significantly higher at respective growth stage when compared with absolute control. 
Table 3: Fresh weight per plant as influenced by different treatments

\begin{tabular}{|c|c|c|c|c|}
\hline \multirow{2}{*}{ Tr. No. } & \multirow{2}{*}{ Treatments } & \multicolumn{3}{|c|}{ Fresh weight per plant (g) } \\
\hline & & 45 DAS & 60 DAS & 75 DAS \\
\hline $\mathrm{T}_{1}$ & Premax + Rizo-liq (15 DPS) & 13.36 & 17.50 & 24.38 \\
\hline $\mathrm{T}_{2}$ & Premax + Rizo-liq (10 DPS) & 13.38 & 17.79 & 26.84 \\
\hline $\mathrm{T}_{3}$ & Premax + Rizo-liq (05 DPS) & 13.84 & 18.41 & 29.09 \\
\hline $\mathrm{T}_{4}$ & Premax + Rizo-liq (01 DPS) & 14.94 & 19.23 & 32.74 \\
\hline $\mathrm{T}_{5}$ & Premax + Rizo-liq (ODS) & 15.69 & 20.62 & 33.76 \\
\hline $\mathrm{T}_{6}$ & Thiram+Carbendazim 50 WP-Premax+Rizo-liq (15 DPS) & 13.68 & 17.77 & 27.72 \\
\hline $\mathrm{T}_{7}$ & Thiram +Carbendazim 50 WP-Premax+Rizo-liq (10 DPS) & 13.98 & 19.13 & 29.96 \\
\hline $\mathrm{T}_{8}$ & Thiram +Carbendazim 50 WP-Premax+Rizo-liq (05 DPS) & 15.05 & 19.28 & 30.97 \\
\hline $\mathrm{T}_{9}$ & Thiram+Carbendazim 50 WP-Premax+Rizo-liq (01 DPS) & 15.61 & 19.66 & 32.08 \\
\hline $\mathrm{T}_{10}$ & Thiram+ Carbendazim 50 WP - Premax + Rizo-liq (ODS) & 16.44 & 22.60 & 35.83 \\
\hline $\mathrm{T}_{11}$ & Thiram + Carbendazim $50 \mathrm{WP}-\mathrm{Rh}_{1}(\mathrm{ODS})$ & 15.67 & 20.52 & 34.19 \\
\hline $\mathrm{T}_{12}$ & Thiram + Carbendazim $50 \mathrm{WP}-\mathrm{Rh}_{2}(\mathrm{ODS})$ & 15.97 & 19.79 & 34.09 \\
\hline $\mathrm{T}_{13}$ & Thiram + Carbendazim $50 \mathrm{WP}-\mathrm{Rh}_{3}(\mathrm{ODS})$ & 16.08 & 21.60 & 34.83 \\
\hline $\mathrm{T}_{14}$ & Uninoculated seed (but fungicidal treatment) & 12.59 & 16.61 & 23.60 \\
\hline $\mathrm{T}_{15}$ & Absolute control (No fungicidal treatment and no inoculation) & 10.57 & 14.58 & 20.20 \\
\hline $\mathrm{SEm} \pm$ & & 0.81 & 1.10 & 1.76 \\
\hline $\mathrm{CD}$ at $5 \%$ & & 2.30 & 3.14 & 5.01 \\
\hline
\end{tabular}

\section{Dry weight per plant (g)}

Data revealed that average dry matter per plant increased gradually with increase in the age of crop. The accumulation was maximum between 60 and 75 DAS in almost all the treatments. The application of $\mathrm{T}_{10}$ [Thiram+carbendazim 50 $\mathrm{W}$ - Premax+ Rizo-liq (ODS)] treatment registered the maximum dry weight per plant followed by $\mathrm{T}_{13}$ [Thiram + Carbendazim $50 \mathrm{WP}-\mathrm{Rh}_{3}$ (ODS)] treatment at all stages. Dry matter per plant at 75 DAS was maximum $(23.14 \mathrm{~g})$ in $\mathrm{T}_{10}$ [Thiram+carbendazim $50 \mathrm{WP}$ - Premax+ Rizo-liq (ODS)] treatment and minimum (14.6 g) in $\mathrm{T}_{1}$ (Absolute control) treatment.

Table 4: Dry weight per plant as influenced by different treatments

\begin{tabular}{|c|c|c|c|c|}
\hline \multirow{2}{*}{ Treatment No. } & \multirow{2}{*}{ Treatments } & \multicolumn{3}{|c|}{ Dry weight per plant (g) } \\
\cline { 2 - 5 } & & 45 DAS & $\mathbf{6 0}$ DAS & $\mathbf{7 5}$ DAS \\
\hline $\mathrm{T}_{1}$ & Premax + Rizo-liq (15 DPS) & 5.44 & 8.65 & 16.15 \\
\hline $\mathrm{T}_{2}$ & Premax + Rizo-liq (10 DPS) & 5.64 & 8.97 & 16.33 \\
\hline $\mathrm{T}_{3}$ & Premax + Rizo-liq (05 DPS) & 5.86 & 9.66 & 16.80 \\
\hline $\mathrm{T}_{4}$ & Premax + Rizo-liq (01 DPS) & 5.92 & 9.88 & 17.75 \\
\hline $\mathrm{T}_{5}$ & Premax + Rizo-liq (ODS) & 6.05 & 9.98 & 20.40 \\
\hline $\mathrm{T}_{6}$ & Thiram + Carbendazim 50 WP - Premax + Rizo-liq (15 DPS) & 5.77 & 8.75 & 17.33 \\
\hline $\mathrm{T}_{7}$ & Thiram + Carbendazim 50 WP - Premax + Rizo-liq (10 DPS) & 5.82 & 8.94 & 17.38 \\
\hline $\mathrm{T}_{8}$ & Thiram + Carbendazim 50 WP - Premax + Rizo-liq (05 DPS) & 5.76 & 9.51 & 18.58 \\
\hline $\mathrm{T}_{9}$ & Thiram + Carbendazim 50 WP - Premax + Rizo-liq (01 DPS) & 6.10 & 9.92 & 19.45 \\
\hline $\mathrm{T}_{10}$ & Thiram + Carbendazim 50 WP - Premax + Rizo-liq (ODS) & 7.02 & 11.01 & 23.14 \\
\hline $\mathrm{T}_{11}$ & Thiram+Carbendazim 50 WP - Rh 1 (ODS) & 6.22 & 10.51 & 21.95 \\
\hline $\mathrm{T}_{12}$ & Thiram+Carbendazim 50 WP - Rh ${ }_{2}$ (ODS) & 6.02 & 9.91 & 20.33 \\
\hline $\mathrm{T}_{13}$ & Thiram+Carbendazim 50 WP - Rh ${ }_{3}$ (ODS) & 6.47 & 10.73 & 22.78 \\
\hline $\mathrm{T}_{14}$ & Uninoculated seed (but fungicidal treatment) & 5.37 & 8.46 & 15.70 \\
\hline $\mathrm{T}_{15}$ & Absolute control (No fungicidal treatment and no inoculation) & 5.05 & 8.07 & 14.60 \\
\hline $\mathrm{SEm} \pm$ & & 0.25 & 0.40 & 1.13 \\
\hline $\mathrm{CD}$ at $5 \%$ & & 0.72 & 1.15 & 3.23 \\
\hline
\end{tabular}

\section{Leaf Area Index (LAI)}

LAI, a parameter of canopy growth was estimated at 30, 40, 60 and 75 DAS. Leaf area index was maximum at 60 DAS. Maximum leaf area index (12.87) at $60 \mathrm{DAS}$ was found in $\mathrm{T}_{10}$ [Thiram+carbendazim 50 WP - Premax+ Rizo-liq (ODS)] treatment and it was significantly superior over other treatments except $\mathrm{T}_{13}, \mathrm{~T}_{5}, \mathrm{~T}_{11}$ and $\mathrm{T}_{9}$ treatments. Minimum leaf area index (10.54) was recorded in $\mathrm{T}_{5}$ (Absolute control) treatment. 
Table 5: Leaf area index as influenced by various treatments at successive stages of plant growth

\begin{tabular}{|c|c|c|c|c|c|}
\hline \multirow{2}{*}{ Treatment No. } & \multirow{2}{*}{ Treatments } & \multicolumn{4}{|c|}{ Leaf area index } \\
\hline & & 30 DAS & 45 DAS & 60 DAS & 75 DAS \\
\hline $\mathrm{T}_{1}$ & Premax + Rizo-liq (15 DPS) & 3.29 & 4.62 & 10.81 & 6.88 \\
\hline $\mathrm{T}_{2}$ & Premax + Rizo-liq (10 DPS) & 3.43 & 4.78 & 10.93 & 7.29 \\
\hline $\mathrm{T}_{3}$ & Premax + Rizo-liq (05 DPS) & 3.58 & 4.99 & 11.02 & 7.4 \\
\hline $\mathrm{T}_{4}$ & Premax + Rizo-liq (01 DPS) & 3.69 & 5.21 & 11.07 & 7.67 \\
\hline $\mathrm{T}_{5}$ & Premax + Rizo-liq (ODS) & 4.32 & 6.16 & 12.15 & 7.97 \\
\hline $\mathrm{T}_{6}$ & Thiram + Carbendazim 50 WP - Premax + Rizo-liq (15 DPS) & 3.55 & 5.24 & 11.05 & 7.48 \\
\hline $\mathrm{T}_{7}$ & Thiram + Carbendazim 50 WP - Premax + Rizo-liq (10 DPS) & 3.65 & 5.31 & 11.05 & 7.67 \\
\hline $\mathrm{T}_{8}$ & Thiram + Carbendazim 50 WP - Premax + Rizo-liq (05 DPS) & 3.73 & 5.55 & 11.26 & 7.74 \\
\hline $\mathrm{T}_{9}$ & Thiram + Carbendazim 50 WP - Premax + Rizo-liq (01 DPS) & 4.09 & 5.78 & 11.96 & 7.94 \\
\hline $\mathrm{T}_{10}$ & Thiram + Carbendazim 50 WP - Premax + Rizo-liq (ODS) & 5.18 & 6.75 & 12.87 & 8.24 \\
\hline $\mathrm{T}_{11}$ & Thiram + Carbendazim 50 WP - $\mathrm{Rh}_{1}(\mathrm{ODS})$ & 4.56 & 6.15 & 11.79 & 7.94 \\
\hline $\mathrm{T}_{12}$ & Thiram + Carbendazim 50 WP - $\mathrm{Rh}_{2}(\mathrm{ODS})$ & 4.62 & 6.06 & 11.70 & 7.91 \\
\hline $\mathrm{T}_{13}$ & Thiram + Carbendazim 50 WP - $\mathrm{Rh}_{3}(\mathrm{ODS})$ & 4.97 & 6.59 & 12.50 & 8.16 \\
\hline $\mathrm{T}_{14}$ & Uninoculated seed (but fungicidal treatment) & 3.19 & 4.66 & 10.75 & 6.73 \\
\hline $\mathrm{T}_{15}$ & Absolute control (No fungicidal treatment and no inoculation) & 3.06 & 4.54 & 10.54 & 6.12 \\
\hline $\mathrm{SEm} \pm$ & & 0.33 & 0.40 & 0.40 & 0.33 \\
\hline $\mathrm{CD}$ at $5 \%$ & & 0.94 & 1.13 & 1.14 & 0.95 \\
\hline
\end{tabular}

\section{Yield attributing characters}

\section{Number of pods per plant}

The maximum (39.18 pods/plant) number of pods in entire experiment was recorded in $\mathrm{T}_{10}$ [Thiram+Carbendazim 50 WP - Premax+ Rizo-liq (ODS)] treatment, which was significantly superior to treatment $\mathrm{T}_{14}$ (Uninoculated seed), $\mathrm{T}_{15}$ (Absolute control) and at par with rest of the treatments. Minimum pods (24.53/plant) were recorded in $\mathrm{T}_{15}$ (Absolute control) treatment. It is evident from the data (Table 6) that Rhizobium inoculation with or without fungicides resulted in significant increase in number of pods per plant as compared to the control and uninoculated seed treatment.

\section{Number of seeds per pod}

Data showed that the there was a variation in seeds per pod but statistically it was non significant. However, the maximum (2.84 seeds per pod) number of seeds per pod in entire experiment was recorded in $\mathrm{T}_{10}$ [Thiram+Carbendazim 50 WP - Premax+ Rizo-liq (ODS)] treatment. Minimum seeds (2.45/pod) were recorded in $\mathrm{T}_{15}$ (Absolute control) treatment.

Table 6: Average number of pods per plant and seeds per pod as influenced by different treatments

\begin{tabular}{|c|c|c|c|}
\hline Treatment No. & Treatments & Number of pods per plant & Number of seeds per pod \\
\hline $\mathrm{T}_{1}$ & Premax + Rizo-liq (15 DPS) & 34.20 & 2.63 \\
\hline $\mathrm{T}_{2}$ & Premax + Rizo-liq (10 DPS) & 34.43 & 2.65 \\
\hline $\mathrm{T}_{3}$ & Premax + Rizo-liq (05 DPS) & 34.63 & 2.68 \\
\hline $\mathrm{T}_{4}$ & Premax + Rizo-liq (01 DPS) & 35.70 & 2.69 \\
\hline $\mathrm{T}_{5}$ & Premax + Rizo-liq (ODS) & 36.80 & 2.74 \\
\hline $\mathrm{T}_{6}$ & Thiram +Carbendazim 50 WP - Premax + Rizo-liq (15 DPS) & 34.55 & 2.66 \\
\hline $\mathrm{T}_{7}$ & Thiram +Carbendazim 50 WP - Premax + Rizo-liq (10 DPS) & 35.43 & 2.68 \\
\hline $\mathrm{T}_{8}$ & Thiram +Carbendazim 50 WP - Premax + Rizo-liq (05 DPS) & 35.48 & 2.7 \\
\hline $\mathrm{T}_{9}$ & Thiram +Carbendazim 50 WP - Premax + Rizo-liq (01 DPS) & 36.23 & 2.7 \\
\hline $\mathrm{T}_{10}$ & Thiram +Carbendazim 50 WP - Premax + Rizo-liq (ODS) & 39.18 & 2.84 \\
\hline $\mathrm{T}_{11}$ & Thiram + Carbendazim 50 WP - $\mathrm{Rh}_{1}(\mathrm{ODS})$ & 37.23 & 2.76 \\
\hline $\mathrm{T}_{12}$ & Thiram + Carbendazim $50 \mathrm{WP}-\mathrm{Rh}_{2}(\mathrm{ODS})$ & 36.55 & 2.74 \\
\hline $\mathrm{T}_{13}$ & Thiram + Carbendazim 50 WP - $\mathrm{Rh}_{3}(\mathrm{ODS})$ & 39.00 & 2.78 \\
\hline $\mathrm{T}_{14}$ & Uninoculated seed (but fungicidal treatment) & 31.13 & 2.6 \\
\hline $\mathrm{T}_{15}$ & Absolute control (No fungicidal treatment and no inoculation) & 24.53 & 2.45 \\
\hline SEm \pm & & 1.96 & 0.07 \\
\hline $\mathrm{CD}$ at $5 \%$ & & 5.59 & NS \\
\hline
\end{tabular}

\section{Seed yield per hectare}

Yield of the crop is the result of the various biotic and environmental factors, which are responsible for changes brought about in the productivity. Effectiveness of any treatment could be judged by the magnitude of changes in the productivity brought about by that particular treatment. The seed yield was recorded per net plot and then it was converted into $\mathrm{kg} / \mathrm{ha}$. Perusal of data in Table 7 revealed that the maximum $(2.15 \mathrm{t} / \mathrm{ha})$ seed yield of soybean in entire experiment was recorded in $\mathrm{T}_{10}$ [Thiram+carbendazim $50 \mathrm{WP}$ - Premax+ Rizo-liq (ODS)] treatment which was appreciably higher than all other treatments, immediately followed by $\mathrm{T}_{13}$ [Thiram + Carbendazim 50 WP - $\mathrm{Rh}_{3}$ (ODS)] (2.12 t/ha) treatment. Minimum $(1.51 \mathrm{t} / \mathrm{ha})$ seed yield was recorded in $\mathrm{T}_{15}$ (Absolute control) treatment.

\section{Stover yield per hectare}

The stover yield obtained was statistically analysed in order to find out the effect of different treatments. The stover yield was calculated by subtracting the seed yield from the biological yield. The maximum $(2.85 \mathrm{t} / \mathrm{ha})$ stover yield in entire experiment was recorded in $\mathrm{T}_{10}$ [Thiram+Carbendazim 50 WP- Premax+ Rizo-liq (ODS)] treatment. $\mathrm{T}_{10}$ [Thiram+Carbendazim 50 WP- Premax+ Rizo-liq (ODS)] was found to be at par with all the treatments except $T_{1}, T_{2}, T_{14}$, and $\mathrm{T}_{15}$ (Absolute control). The lowest stover yield $(2.08 \mathrm{t} / \mathrm{ha})$ was noted in $\mathrm{T}_{15}$ (Absolute control) treatment. 


\section{Harvest index (\%)}

Data revealed that all the treatments increased the harvest index of soybean as compared to $\mathrm{T}_{15}$ (Absolute control) treatment. The differences in harvest index among the treatments were non significant. The maximum harvest index up to $43.62 \%$ equally found in $\mathrm{T}_{11}$ [Thiram + Carbendazim 50 $\left.\mathrm{WP}-\mathrm{Rh}_{1}(\mathrm{ODS})\right]$ and $\mathrm{T}_{1}$ [Premax+Rizo-liq (15 DPS)] treatments whereas the minimum harvest index (39.56\%) was recorded in $\mathrm{T}_{15}$ (Absolute control).

Table 7: Seed yield, Stover yield (tonne/ha) and harvest index (\%) as affected by different treatments

\begin{tabular}{|c|c|c|c|c|}
\hline Treatment No. & Treatments & $\begin{array}{c}\text { Seed Yield } \\
(\mathbf{t} / \mathbf{h a})\end{array}$ & $\begin{array}{c}\text { Stover } \\
\text { Yield (t/ha) }\end{array}$ & $\begin{array}{c}\text { Harvest } \\
\text { Index (\%) }\end{array}$ \\
\hline $\mathrm{T}_{1}$ & Premax + Rizo-liq (15 DPS) & 1.85 & 2.39 & 43.61 \\
\hline $\mathrm{T}_{2}$ & Premax + Rizo-liq (10 DPS) & 1.85 & 2.42 & 43.33 \\
\hline $\mathrm{T}_{3}$ & Premax + Rizo-liq (05 DPS) & 1.90 & 2.54 & 42.84 \\
\hline $\mathrm{T}_{4}$ & Premax + Rizo-liq (01 DPS) & 1.99 & 2.61 & 43.43 \\
\hline $\mathrm{T}_{5}$ & Premax + Rizo-liq (ODS) & 2.06 & 2.73 & 43.32 \\
\hline $\mathrm{T}_{6}$ & Thiram+Carbendazim 50 WP-Premax + Rizo-liq (15 DPS) & 1.90 & 2.53 & 43.00 \\
\hline $\mathrm{T}_{7}$ & Thiram+Carbendazim 50 WP-Premax + Rizo-liq (10 DPS) & 1.94 & 2.65 & 42.15 \\
\hline $\mathrm{T}_{8}$ & Thiram+Carbendazim 50 WP-Premax + Rizo-liq (05 DPS) & 1.98 & 2.65 & 42.69 \\
\hline $\mathrm{T}_{9}$ & Thiram+Carbendazim 50 WP-Premax + Rizo-liq (01 DPS) & 2.02 & 2.67 & 43.37 \\
\hline $\mathrm{T}_{10}$ & Thiram+Carbendazim50 WP-Premax + Rizo-liq (ODS) & 2.15 & 2.85 & 43.20 \\
\hline $\mathrm{T}_{11}$ & Thiram + Carbendazim 50 WP - Rh (ODS) & 2.03 & 2.63 & 43.62 \\
\hline $\mathrm{T}_{12}$ & Thiram + Carbendazim 50 WP - Rh 2 (ODS) & 1.93 & 2.72 & 41.42 \\
\hline $\mathrm{T}_{13}$ & Thiram + Carbendazim 50 WP - Rh 3 (ODS) & 2.12 & 2.76 & 43.36 \\
\hline $\mathrm{T}_{14}$ & Uninoculated seed (but fungicidal treatment) & 1.64 & 2.17 & 43.03 \\
\hline $\mathrm{T}_{15}$ & Absolute control (No fungicidal treatment and no inoculation) & 1.51 & 2.08 & 39.56 \\
\hline SEm \pm & & 0.07 & 0.13 & 1.67 \\
\hline CD at 5\% & & 0.21 & 0.36 & $\mathrm{NS}$ \\
\hline
\end{tabular}

DPS: Days prior sowing, ODS: On days of sowing, DAS: Days after sowing

\section{References}

1. Ahiabor B, Lamptey S, Yeboah S, Bahari V. Application of phosphorus fertilizer on soybean [(Glycine max L. (Merril)] inoculated with rhizobium and its economic implication to farmers. American Journal of Experimental Agriculture. 2014; 4(11):1420-1434.

2. Barik KC, Chandel AS. Effect and phosphorus uptake in soybean (Glycine max) and their residual availability in Mallisol. Indian Journal of Agronomy. 2001; 46(2):319326.

3. Bhattarai HD, Prasad BN. Effect of dual inoculation of Bradyrhizobium japonicum and Azotobactor chroococcum. Indian Journal of Microbiology. 2003; 43(2):139-140.

4. Chendrayan OK, Natrajan T, Umamaheshwari $T$. Combined inoculation of Bio fertilizers for increasing crop production. Biofertilizer Newsletter. 2003; 11(2):2426.

5. Dadson RB, Acquash G. Rhizobium japonicum, nitrogen, phosphorus effects on nodulation, symbiotic fixation and yield of soybean. Field Crop Research. 1984; 9(2):101108.

6. Dubey SK. Increasing efficacy of Phosphatic fertilizers through bio-inoculation of Bradyrhizobium japonicum and phosphate solubilizing bacteria in rainfed soybean (Glycine max) Journal Oilseeds Research. 2003; 20(1):149-152.

7. Gautam P, Agnihotri AK. Economic feasibility of Bradyrhizobium japonicum, farm yard manure and pseudomonas sp. with phosphorus in soybean. Indian farming. 2005, 11-26.

8. Govindan K, Thirumurugan. Effect of Rhizobium and PSM's in Soybean. Journal of Maharashtra Agricultural University. 2003; 28(1):54-60.

9. Kolhapure DJ, Memane SA, Rasal PH, Pawar KB. Varietal response of soybean to different strains of Bradyrhizobium japonicum. Journal of Maharashtra Agricultural University. 2003; 28(2):161-163.
10. Kumar S, Upadhyay JP, Roy S, Kumar S. Effect of pesticide seed dressing and Rhizobium inoculation on nodulation and yield of chickpea (Cicer arietinum). Journal of Applied Biology. 2002; 12(1-2):81-83.

11. Kumari S, Mahapatra P, Shahi DK, Singh AK. Effect of $\mathrm{P}, \mathrm{S}$ and Bradyrhizobium on yield, nutrient content and uptake by soybean (Glycine max) under rainfed condition. Journal of Pharmacognosy and Phytochemistry. 2019; 8(6):2554-2557

12. Menaria BL, Singh P. Effect of NPK and S combinations and microbial inoculants on nodulation, yield and N,P content of soil after harvest of soybean (Glycine max (L.) Merrill). Annals of Agricultural Research New Series. 2004; 25(1):162-163.

13. Meshram SU, Pande SS, Shavre AS, Kamdi RR, Tajane VS. Efficacy of Biofertilizers integrated with chemical fertilizers in vivo in soybean. Biofertilizer Newsletter. 2004; 12(1):7-10.

14. Osei D, Lamptey S, Ayisi CL, Apraku A. Effects of rhizobium inoculants and growth stages on shoot biomass and yield of soybean (Glycine $\max (\mathrm{L}$.$) merril).$ International Journal of Scientific and Technology Research. 2014; 3(4):321-327.

15. Rajeshwari MC, Hunje R, Badiger BA, Jahagirdar S. Influence of fungicides and bioagents seed treatment on seed yield and quality in soybean [Glycine max (L.) Merrill]. International Journal of Chemical Studies. 2020; 8(2):2261-2264

16. Singh R, Rai RK. Yield attributes, yield and quality of soybean (Glycine max) as influenced by integrated nutrient management. Indian Journal of Agronomy. 2004; 49(4):271-274.

17. Tyagi MK, Bhattacharya $\mathrm{P}$, Yadav AK. Effect of Rhizobium and phosphorus solubilizing bacteria on the yield of Pea (Pisum sativum). Biofertilizer Newsletter. 2004; 12(2):9-14. 